\title{
Personalized and Precision Medicine (PPM) as a Unique Healthcare Model of the Future to Come: Hype or Hope?
}

\author{
Maria Studneva $^{1 *}$, Anton Yuryev ${ }^{9}$, Veronica Medvedeva ${ }^{1}$, Hiroyuki Abe ${ }^{10}$, Elena Antonova ${ }^{1,8}$, Vassiliy Tsytsarev ${ }^{11,12}$, Trevor Marshall ${ }^{7}$, \\ Andrew D Miller ${ }^{12-15}$ and Sergey Suchkov ${ }^{1-6}$
}

'Sechenov University, Moscow, Russia

${ }^{2}$ A.I.Evdokimov Moscow State University of Medicine \& Dentistry, Russia

${ }^{3}$ EPMA, Brussels, EU, Belgium

${ }^{4} \mathrm{PMC}$, Washington, DC, USA

${ }^{5}$ ISPM and Abe Cancer Clinic, Tokyo, Japan

${ }^{6} \mathrm{AHA}$, Houston, TX, USA

${ }^{7}$ Autoimmunity Research Foundation, Los Angeles, CA, USA

${ }^{8}$ Municipal N.F.Filatov's Cinical Pediatric Hospital, Russia

${ }^{9}$ Center for Bioinformatics, Elsevier, Rockville, MD, USA

${ }^{10} \mathrm{ISPM}$, Tokyo, Japan

${ }^{11}$ Fischell Center of Bioengineering, University of Maryland, College Park, USA

${ }^{12}$ Dept for Neuronal Networks, John Hopkins University, MD, USA

${ }^{13}$ Veterinary Research Institute and Mendel University, Brno, Czech Republic

${ }^{15}$ Shanghai Jiao Tong University, Shanghai, PRC

${ }^{\star}$ Corresponding author: Maria Studneva, Sechenov University, Moscow, Russia

Received: November 25, 2020; Accepted: December 08, 2020; Published: December 14, 2020

\begin{abstract}
A new systems approach to diseased states and wellness result in a new branch in the healthcare services, namely, Personalized and Precision Medicine (PPM). To achieve the implementation of PM concept, it is necessary to create a fundamentally new strategy based upon the subclinical recognition of biopredictors of hidden abnormalities long before the disease clinically manifests itself.

Each decision-maker values the impact of their decision to use PPM on their own budget and well-being, which may not necessarily be optimal for society as a whole. It would be extremely useful to integrate data harvesting from different databanks for applications such as prediction and personalization of further treatment to thus provide more tailored measures for the patients resulting in improved patient outcomes, reduced adverse events, and more cost effective use of health care resources. A lack of medical guidelines has been identified by the majority of responders as the predominant barrier for adoption, indicating a need for the development of best practices and guidelines to support the implementation of PPM!

Implementation of PPM requires a lot before the current model «physician-patient» could be gradually displaced by a new model «medical advisorhealthy person-at-risk». This is the reason for developing global scientific, clinical, social, and educational projects in the area of PPM to elicit the content of the new branch.
\end{abstract}

Keywords: Translational research, Personalized \& precision medicine (PPM), Next-generation sequencing (NGS), Drug discovery, Educational cluster, Education-scienceinnovation complexes (ESIC)

\section{Introduction}

Translational Research \& Applications (TRA) is a term used to describe a complex process aimed to build on basic scientific research to create new therapies, medical procedures and diagnostics [1]. It is critical that scientists are well acquainted with organogenesis and of human pathogenesis arising from microbial infection and natural errors in gene functioning. Most students and scientists do not have sufficient knowledge.
Medical scholars and students are acquainted with basic anatomy and even basic molecular biology, but their under-standing of fundamental processes located in living systems is limited. For instance, the concept of "stem cell" in human development and in pathogeneses as in the development of cancers or atherosclerotic plaque are not yet understood even in the world's leading centers of basic and medical research.

Despite a tremendous impact of human genome project on our understanding of the pathogenesis of cancer, autoimmune and other 
chronic conditions and an invention of different techniques such as single cell sequencing or proteome and metabolome profiling, the current educational system is not opened and thus sufficient at preparing a next-generation specialist, which is able to use all the advances have been made [2]. For example, an implementation of NGS into clinical practice requires a "Big data" approach based on high integrity between clinical informatics, bio-informatics, and fundamental studies as well to secure finally the proper clinical decision being evidence-based. A major challenge in the clinical setting is the need to support a dynamic work-flow associated with the constant growth of the laboratory's NGS test menu and expanding specimen volume [3]. To perform such kind of the mission it is crucial to educate specialists, which will know medical, biological and informatics aspects of the problem and will know how to use their knowledge in solving this problem as well. And that is just the tip of the iceberg. Human genetic databases are corrupted by false results. NGS studies have built in error rates of approximately 0.3 to $3.0 \%$ per base pair, which does not favor an improved understanding of diseases and the implementation of advanced therapeutics. The concept of Personalized \& Precision Medicine (PPM) requires high integrity between fundamental research, industry, and clinic $[4,5]$.

The lack of translation is the challenging problem in the various fields of medicine such as creating of human-computer interfaces or investigations of drug resistance and cancer [6-8]. We are not saying that the problem is mainly due to the obsolete education system, for example, the lack of clinical translation in cancer research can be explained by the fact that animal models is not a precise reflection of a human organism [8]. Conversion of research findings into meaningful human applications, mostly as novel remedies of human diseases, needs progress of appropriate animal models. Research methodologies to test new drugs in preclinical phases often demanded animal models that not only replicate human disease in etiological mechanisms and pathobiology but also biomarkers for early diagnosis, prognosis, and toxicity prediction. Whereas the transgenic and knockout procedures have developed guidance of rodents and other species to get greater understandings of human disease pathogenesis, but still generating perfect animal models of most human disease is not available [8].

Clinical trials themselves have limitations, and hence the results of these studies could be misunderstood [9]. The point is: in order to provide an effective «bench to bed» workflow there is a huge need for specialists, which are capable of performing a wide range of tasks. Nowadays due to a tremendous amount of available information, it is feasible to create a specialist who knows how to interconnect different areas of research and how to adapt to constantly changing conditions, whereas to create a specialist knowing how to do every-thing on his own is not. An education of these specialists is the pivotal objective for the new education system, and the creation of this system is at the top of the agenda for this paper.

Drug discovery is extremely both time- and money-consuming process. The basic translational pipeline here consists of at least eight units, namely, target to hit, hit to lead, lead optimization, preclinical trials, three stages of clinical trials, and finally, submission to launch [10]. The whole process lasts as long as a decade and a half and requires interdisciplinary-educated staff not only familiar with fundamental research but also with different techniques and approaches used in the drug discovery. A number of potential solutions to improve R\&D productivity and increase clinical translation of drug candidates have been offered by Paul et al. [10]. Some of these solutions propose a total transformation of the current single company-owned R\&D enterprise to one that is highly networked, partnered and leveraged (Fully Integrated Pharmaceutical Network or FIPNet) [10]. Authors also stated that in order to improve drug development it is vital to provide a cash-flow from the high expensive phase II and III trials to less expensive preclinical and 1-st phase clinical trials, thereby increasing the number of drug candidates to select the most promising ones. These candidates, in turn, would have a higher chance to be approved [10]. Obviously, both of the ideas aforementioned require a strong collaboration between research, stakeholders, and government. And we suggest that the education is the starting point to deploy such a network. Once developed, this new education system should kill two birds with one stone, namely, should prompt the collaboration between fundamental research and industry, yet also should allow to use an approach close to simulation-based medical education that has been proved to be highly effective in different areas of medical education [11-15]. The main difference of the approach proposed is the use of it in the settings of drug discovery.

Mastery learning is another approach, which could be used in the modern education system. Despite its development as early as in 1963, it has a lot of progressive features, such as clear learning objectives, deliberate skills practice, and complete mastery of the discipline selected [16]. However, it also has some considerable limitations especially meaningful in a case of drug development. One of these limitations is the unlimited time to reach the mastery. The time factor is one of the most important ones during the translation process. To reduce the negative influence of unlimited time of mastering one could involve only talented students in such a program, which expected time to acquire a new skill or master a new subject is relatively low. However, does it fit in with a standard mastery learning paradigm?

The problem of great concern is the designing of a mechanism that could detect, educate, and implicate highly motivated students and young scientists in order to meet the needs from industry and healthcare system. It is clear that somehow, we should tightly interconnect different areas of research, saving student-oriented education principles. Some attempts are already ongoing: universities are experimenting with new programs and courses to teach innovation. Within the life sciences, there is particularly strong traction in the area of biomedical technology innovation, in which a number of interesting new training initiatives are being developed and deployed. However, how this experiments will affect the healthcare system remains to be determined. System itself requires not just new courses and programs, but a total rearrangement at all.

\section{Fundamental Aspects of the Educational Reforms}

At the present stage, the main task is the development of the concept changes of Healthcare Service and creation of new medical education model. The purpose of employment of the knowledge is to predict and 
prevent diseases, increase the life expectancy, strengthen and preserve human health, as well as the identification and monitoring patients with underlying risk for the development of a particular pathology.

A key reason for changing the health care system became an active use in the practice of a hospital physician of advances in omics, allowing penetrating inside biostructures and creating therein conditions for visualization of lesions, previously concealed from the eyes of a clinician.

At the heart of the developed concept of PPM use there are postulates which promote change in the culture and the mindset of society as a whole. In the first place it is the awareness of individuals that they are responsible for their own health and the health of their children, an active involvement of the people in a sphere of preventive and prophylactic measures designed for promotion of individual, community-related and public health, in particular.

Meanwhile, putting PPM-tools in a public health perspective requires an apprehension of the current and future public health challenges. Those challenges are produced by the new technological developments, health transition, and the increased importance of non-communicable diseases, even in low-income environments.

The principles of PPM and efforts to approaching the right health issues in a timely manner can be applied to public health. Doing so will, however, require a careful view and concerted effort to maintain the needs of public health at the forefront of all PPM discussions and investments. Briefly, a prime concern for public health is promoting health, preventing disorder, and reducing health disparities by focusing on modifiable morbidity and mortality. In this connection, more-accurate and precise methods for measuring disease, pathogens, exposures, behaviors, and susceptibility could allow better assessment of public and individual health and development of policies and targeted programs for preventing disease and managing disorders at the individualized level whilst operating with precision tools and datasets. So, the initial drive toward PPM-based public health is occurring, but much more work lies ahead to develop a robust evidentiary foundation for use.

In this connection, one of the major organizational tasks is to carry out restructuring of the existent health care system to ensure implementation of preventive, diagnosis, remedial and rehabilitation measures designed to reduce morbidity and death rate of population, ensure maternal and infant health care and promote healthy lifestyle.

Implementation of the PPM model will lead to the replacement of the existing "doctor-patient" relationship model by the "doctorconsultant-healthy person" model. In this regard, it is obvious that the society needs a new scientific and technical school for the formation of specialists of a new generation, using non-traditional methods and a technological arsenal based on the achievements of systems biology and translational medicine.

For training of specialists it is required to restructure programs of pre-university, undergraduate, graduate and postdoctoral medical training as well as to develop fundamentally new interdisciplinary programs, focused on training of specialists in the areas related to PPM. In implementing the principle of continuity of an ongoing education a model of multi-stage training of a specialist is being built, which is characterized by a phase-by-phase process of individual development going over, while information is learned, from one level of an ongoing training to another.

In such a manner, at the 1st level of education (pre-university) school special significance is on the pre-university level is the selection of talented young specialists and involvement them into creative activities. At the $2^{\text {nd }}$ (university) level, students will be offered in-depth study of fundamental and applied aspects of PPM. The core of the third (post-university) level will be interdisciplinary aspects of PPM, targeted to resident physicians and postgraduates [17-21].

An important component of the new educational model is its focus on the practical skills and the ability to apply knowledge. Many of universities has already organized ESIC for the purpose of increasing the quality of education and strengthening the liaison with the production. The specifics of ESIC consists in that thanks to the cooperation of scientific research, educational and production capacities, there is ensured a new quality of education, development of research and commercialization of the results of scientific and technological joint performance.

\section{Some Features of the Educational Model}

Currently, the first roots of the new educational program are being developed within the heart of the Russian medical community, aimed at training doctors and specialists in the field of biopharma. Within the program it is planned to train specialists for medical, pediatric and bioengineering faculties. The courses of the program are divided into three categories, i.e., basic, elective and specialized. At the first stage of pre-university training, general aspects of human physiology and anatomy, the foundations of molecular and cell biology will be considered, and also students will learn the basics of PPM. The first includes the first two courses, in which students will study the fundamental foundations of PPM (Omics, Genetic Engineering, Genomic Editing and Gene Therapy, Immunology, Biomarkers, Bioinformatics, Targeting, Technologies for Working with Proteins and Genes, Biobanks). Further, at the stage of the three-year university education will be the study of diagnostic, preventive and therapeutic diagnostic platforms of target categories of PPM, among them pharmacogenomics, oncology, pulmonology, pediatrics and others. At the next one-year training stage, students will study clinical and preclinical models with predictive-diagnostic and preventivepreventive orientation, risks, their evaluation and the formation of diagnostic protocols. At the postgraduate stage students will study preclinical and clinical trials using the biobank base, a program for managing one's own health, including family planning, the stage of genomic scanning and clinical evaluation, clinical bioinformatics, as well as interdisciplinary aspects, including bioethics, the basis of public-private partnerships in modeling personalized and preventive medicine and questions of sociology.

An important part of program is the creation and development of fundamentally new technological platforms with elements the 
commercialization of the results of basic research and following introduction of them into clinical practice. For example, the development of innovative methods system of screening and monitoring will allow estimating the reserves of health, allocate among the asymptomatic contingent in the process of preventive examinations of patients and persons from risk groups with preclinical stages, and create objective prerequisites for personalized therapy. And the creation of an information system for personalized medicine prescribes the development of a new model of the patient and people at risk with using biomarkers, preclinical and predictive diagnostics technologies, and the development of new methods for targeting and motivating healthy lifestyles and active longevity. The key to implementing PPM in clinical practice is information technologies, including machine learning and artificial intelligence.

\section{Obstacles and Problems to Battle Seems to Hamper the Implementation}

World practice has shown that as soon as a country enters a phase of sustainable economic development, there is an increase in the social welfare of people and an increase in the life expectancy of the population, then at the same time an increase in the death rate of the population from cancer and cardiovascular diseases is observed. The priority struggle against socially significant ills of modern civilization is an important step, but it is not decisive in increasing the life expectancy of the population of the country. In the civilized world, there was a steady idea of how to fundamentally reverse the negative trend of growth of socially significant diseases without financial bleeding of the country's budget. More and more economically developed countries are converting their health care in line with the concept of PPM.

Changing the paradigm of health care actually entails reformatting the system for training specialists, reorienting research centers to solving health problems and creating new breakthrough technologies, and qualitatively modernizing the domestic bio-pharmaceutical industry and related industries in the Russian Federation. It is obvious that without interactive regulation and restriction of "egoistic" requests of departments, participants of this global project, any financial investments only in health care and education will be ineffective.

The implementation of the project to modernize health care in its scientific, technical and social significance is akin to a nuclear project of the USSR. Its result was not only the emergence of the country's "atomic shield", but also the creation of new knowledge-intensive branches of the national economy, which ensured economic progress and improved well-being of citizens. The PPM project is aimed at preserving and improving the quality of health of those who are protected by the "atomic shield" of the country. Taking into account the modern structure of the Russian economy, as well as the role of the state in regulating financial flows in the implementation of projects of such scale, it is necessary to give it a special status with the involvement of all possible sources of financing for its implementation.

If we consider the modernization of education as an element of the project with modern scientific and technical achievements, then we have a chance to transform the educational system taking into account breakthrough precision technological platforms. At the same time, in the very system of today's education, there are yesterday's mechanisms that inhibit its mobility and ability to reform.

Despite an ample need to Implementing new educational system into practice, there are some considerable limitations, which could hamper all the process.

First of all, how should we evaluate the impact of the reform on national health care system, quality of life, and even the employment of biopharma specialists? And, in the case of failure, what actions should be performed to prevent additional aggravations in the industry? The main issue here is that we don't have an approach to a transparent analysis of such the data. For instance, a social return on investment - based approach seems to be a promising one due to the fact that it includes the information on the amounts of resources used by a program, in addition to program activities, and represent program value to society as a whole rather than a specific stakeholder group. Unfortunately, this approach is not devoid of flaws, such as raw methods in use and the possibility of inclusion only "appropriate" social groups in the analysis [21,22]. Additionally, several years (or even decades) should pass to enrich the data of reform outcome, and thus allowing to analyze the impact of this reform.

The second issue is the cost of the reform in a broad sense. When speaking of a total rearrangement of the educational system, it is of great importance to determine the source of financing. It seems to be obvious that both the government and the industry are interested in a new education system. However, are these sides interested enough to provide an immense amount of investments required to reach this goal, taking into account that return on investment is not expected in upcoming years? Moreover, to make the reform real it is crucial to implicate well-qualified staff, which demand a salary at least higher than average. Increased administrative expenses, expenses on reform implication, wages for workers, and on additional factors, such as new equipment, could eventually increase the cost of undergraduate and graduate education.

And finally, the educational reform is multifaceted, timeconsuming process, which could be viewed as a process with its own translation pathway. Taking into account that calls for reform of graduate medical education started as early as in 1940 [18], and nothing has changed dramatically ever since (in terms of the education system), the major issue is to prevent the reform from getting stuck in the translation.

\section{Conclusion}

Health care today is in crisis as it is reactive, inefficient, and focused largely on one-size-fits-all treatments for events of late-stage disease. An answer is PPM which is Benefitting patients across many different diseases and even persons-at-risk to prevent a state of being diseased!

The first wave of PPM has entered mainstream clinical practice and is changing the way many diseases are identified, classified, and 
treated. The second wave (a wave of targeted therapies) has turned lots of chronic disorders from deadly ones into the states and conditions in which patients or persons-at-risk live close to normal life spans. In this sense, biopharma and biotech are becoming committed to advancing PPM-related armamentarium, and, in turn, the research and development pipeline would secure great promise for targeted therapies.

So, PPM can create efficiencies in the health care system as a whole. And to help the latter, partnerships and collaborative alliances would transform the research and development of PPMrelated resources. And, especially, to increase recognition from both government and private stakeholders of the value and promise of PPM whilst resuscitating the policymaker interest for being grown. Since PPM is increasingly becoming an integral part of daily clinical care and we expect this trend to continue along with greater recognition of the value of PPM by payers and providers.

Despite the tremendous advances that have been made to date, much work is needed to further stimulate innovations in PPM. As you might see from the above-mentioned, PPM and PPM-based public health calls for an upgraded approach to support safe and effective deployment of the new enabling predictive, diagnostic and therapeutic technologies not to treat but to get cured!!! This approach (PPM and PPM-based public health) mentioned should be based on postulates which will change the incarnate culture and social mentality! And thus the above-mentioned PPM and PPM-based public health model would strongly need for novel training since the society is in bad need of large-scale dissemination of novel systemic thinking and minding. And upon construction of the new educational platforms in the rational proportions, there would be not a primitive physician created but a medical artist to be able to enrich flow-through medical standards with creative elements to gift for a patient a genuine hope to survive but, in turn, for a person-at-risk - a trust for being no diseased. So, the Grand Change and Challenge to secure our individual, communityrelated and public health and wellness are rooted not in Medicine, and not even in Science! Just imagine WHERE?! In the upgraded Hi-Tech Culture! To secure the next-step outcome in the therapeutic future to secure Prevention, Prophylaxis, Canonical Treatment and Rehabilitation as the New Entity!

Our model for accelerated development of continuous vocational education in the sphere of biopharmaceutics and biopharmaceutical industries is based on the combinatorial approaches (competence, module, personality-activity, program-design and problem-oriented) to the elucidation of innovative processes of modernization of the existing system. Correspondingly, the unit to build up the content of educational programs and sites is the task of pedagogics oriented for the innovation context in education development, and it allows each hearer to organically combine individual and group work with the aim to enrich oneself with the experience of the colleagues, and also to use own professional experience.

The aforestated reform of bio-pharmaceutical education, when implemented, will provide the ability to attain and maintain a professional standard of training for specialists in Russian universities, which in turn, will bring them up to world standards and promote academic, professional and inter-regional mobility. It will also enable the creation of an open system of university education, which will ensure that specialists are well enough trained to work in a constantly changing environment.

\section{References}

1. Dougherty D, Conway PH (2008) The "3T's" road map to transform US health care: the "how" of high-quality care. JAMA 299: 2319-2321. [crossref]

2. Nelson EA, McGuire AL (2010) The need for medical education reform: genomics and the changing nature of health information. Genome Med 2: 18. [crossref]

3. Roy S, LaFramboise WA, Nikiforov YE, Nikiforova MN, Routbort MJ, et al. (2016) Next-Generation Sequencing Informatics: Challenges and Strategies for Implementation in a Clinical Environment. Arch Pathol Lab Med 140: 958-975. [crossref]

4. Bodrova TA, Kostyushev DS, Antonova EN, Slavin S, Gnatenko DA, et al. (2012) Introduction into PPPM as a new paradigm of public health service: an integrative view. EPMA J 3: 16. [crossref]

5. Lemke HU, Golubnitschaja O (2014) Towards personal health care with modelguided medicine: long-term PPPM-related strategies and realisation opportunities within 'Horizon 2020'. EPMA J 5: 8. [crossref]

6. Serruya MD (2014) Bottlenecks to clinical translation of direct brain-computer interfaces. Front Syst Neurosci 8: 226. [crossref]

7. Wijdeven RH, Pang B, Assaraf YG, Neefjes J (2016) Old drugs, novel ways out: Drug resistance toward cytotoxic chemotherapeutics. Drug Resist Updat 28: 65-81. [crossref]

8. IWMak, Evaniew N, Ghert M (2014) Lost in translation: animal models and clinical trials in cancer treatment. Am J Transl Res 6: 114-118. [crossref]

9. Mann DL, Mochly-Rosen D (2013) Translational medicine: mitigating risks for investigators. Nat Rev Drug Discov 12: 327-328. [crossref]

10. Paul SM, Mytelka DS, Dunwiddie CT, Persinger CC, Munos BH, et al. (2010) How to improve R\&D productivity: the pharmaceutical industry's grand challenge. Nat Rev Drug Discov 9: 203-214. [crossref]

11. Lopreiato JO, Sawyer T (2015) Simulation-based medical education in pediatrics. Acad Pediatr 15: 134-142. [crossref]

12. McGaghie WC, Issenberg SB, Cohen ER, Barsuk JH, Wayne DB (2012) Translational educational research: a necessity for effective health-care improvement. Chest 142: 1097-1103. [crossref]

13. Kalaniti K, Campbell DM (2015) Simulation-based medical education: time for a pedagogical shift. Indian Pediatr 52: 41-45.

14. Kim J, Park JH, Shin S (2016) Effectiveness of simulation-based nursing education depending on fidelity: a meta-analysis. BMC Med Educ 16: 152. [crossref]

15. Abdelshehid CS, Quach S, Nelson C, Graversen J, Lusch A, et al. (2013) High-fidelity simulation-based team training in urology: evaluation of technical and nontechnical skills of urology residents during laparoscopic partial nephrectomy. J Surg Educ 70: 588-595. [crossref]

16. McGaghie WC (2015) Mastery learning: it is time for medical education to join the $21^{\text {st }}$ century. Acad Med 90: 1438-1441. [crossref]

17. Yates BT, Marra M (2017) Social Return On Investment (SROI): Problems, solutions ... and is SROI a good investment? Eval Program Plann 64: 136-144.

18. Ludmerer KM (2012) The history of calls for reform in graduate medical education and why we are still waiting for the right kind of change. Acad Med 87: 34-40. [Crossref]

19. Strategy of Development of Medical Science in RF for a Period till 2025.

20. Collection of Educational Programs, Typical Tasks and Issues. Training aid for bachelors as per direction 550800 "Chemical Technology and Biotechnolo-gy» // Shvets VI. [et al.] - Moscow: MV. Lomonosov Moscow State Acade-myof Fine Chemical Technology, 2002. - 2.4 printer's sheet. 
Maria Studneva (2020) Personalized and Precision Medicine (PPM) as a Unique Healthcare Model of the Future to Come: Hype or Hope?

21. Engineering Fundamentals of Biotechnology. Training aid for students of Higher Engineering School [Electronic learning resource]: [interactive training aid]. I Registration Certificate No. 1195 of 14 November 2001. Number of state registration 0320100382; edited by D.G. Pobedimsky.
22. Studneva M, Mandrik M, Song Sh, Tretyak E, Krasnyuk I, et al. (2015) "Strategic aspects of higher education reform to cultivate specialists in diagnostic and biopharma industry as applicable to Predictive, Preventive and Personalized Medicine as the Medicine of the Future", The EPMA Journal 6: 18. [crossref]

\section{Citation:}

Studneva M, Yuryev A, Medvedeva V, Abe H, Antonova E, et al. (2020) Personalized and Precision Medicine (PPM) as a Unique Healthcare Model of the Future to Come: Hype or Hope? Internal Med Res Open J Volume 5(4): 1-6. 